\title{
Creatine in health, medicine and sport: an introduction to a meeting held at Downing College, University of Cambridge, July 2010
}

\author{
Roger Harris
}

(C) Springer-Verlag 2011

In July 2010, a focus group meeting on Creatine in Health, Medicine and Sport was held in the New Howard Theatre, Downing College, University of Cambridge. The last such meeting was held just over 10 years ago at which time the main topic of discussion was the increasing use of creatine (Cr) supplements in sport. While still extensively used by athletes, Cr supplementation (Hultman et al. 1996) is today being examined for its health and medical benefits. The purpose of the Downing College meeting was to bring together leading biochemists and physiologists with those involved with $\mathrm{Cr}$ applications in health, medicine and sport, and to exchange experience and knowledge in this emerging field. Theoretical considerations were given equal weightage to applied applications since an understanding of the basic biochemistry and physiology inevitably will underpin any nutritional, health and medical benefits. Sadly, the teaching of the creatine-phosphorylcreatine-creatine kinase $(\mathrm{Cr} / \mathrm{PCr} / \mathrm{CK})$ system, particularly in the field of sports sciences, is often so simplified these days as to obscure mechanisms supporting, as well as failing, cell functions. From a total of 16 review and 9 research presentations at the Downing College meeting, 12 of the reviews are included in this special issue of Amino Acids.

Creatine is a member of the guanidino phosphagen family, which is unique to eukaryotic cells. Altogether, there are eight related forms, of which seven are considered to be chemically derived from arginine, with arginine itself making up the eighth. As with the others, $\mathrm{Cr}$ may bond a phosphoryl group to form $\mathrm{PCr}$, through the action of CK. The $\mathrm{Cr} / \mathrm{PCr} / \mathrm{CK}$ system has its counterparts in the

R. Harris $(\bowtie)$

Junipa Ltd, Newmarket, UK

e-mail: junipa@ymail.com remaining seven other phosphagens, for instance for arginine (A) there is a phosphorylated arginine (PA) formed through the action of arginine kinase (AK). Since A/PA/ $\mathrm{AK}$ is confined to invertebrates and $\mathrm{Cr} / \mathrm{PCr} / \mathrm{CK}$ is the only form found in vertebrates, it was at one time thought that CK evolved after AK, although today, it is known that both are equally ancient.

The classical role of $\mathrm{PCr}$ is seen as a reservoir of highenergy phosphates defending cellular ATP levels under anaerobic conditions, high rates of energy transfer or rapid fluctuations in energy requirement. Although the high concentration of $\mathrm{PCr}$ in glycolytic fast-twitch fibers supports the role of $\mathrm{PCr}$ as a buffer of ATP, the primary importance of the $\mathrm{CK}$ reaction may in fact be to counteract large increases in ADP, which could otherwise inhibit cellular ATPase mediated systems (Sahlin and Harris 2011). Progressive failure in muscle of the $\mathrm{Cr} / \mathrm{PCr} / \mathrm{CK}$ system to maintain ADP homeostasis may point to a common mechanism of fatigue with glycogen depletion and declining $\mathrm{pHi}$, as well as the onset of adenine nucleotide degradation first shown in human muscle by Hultman et al. (1967).

From examination of the dynamics and distribution of $\mathrm{CK}$, it is evident that the $\mathrm{Cr} / \mathrm{PCr} / \mathrm{CK}$ system contributes to cellular metabolism in at least three fundamental ways; as (1) an immediately available temporal energy buffer at sites of rapid ATP turnover, (2) a spatial energy buffer or intracellular energy transport system (i.e., the $\mathrm{CK} / \mathrm{PCr}$ energy shuttle or circuit), and (3) as a metabolic regulator of oxidative phosphorylation (Guzun et al. 2011; Wallimann et al. 2011). As a temporal buffer, $\mathrm{Cr} / \mathrm{PCr} / \mathrm{CK}$ both regenerates ATP and maintains ADP at a low concentration. The CK/PCr energy shuttle connects sites of ATP production (glycolysis and mitochondrial oxidative phosphorylation) with subcellular sites of ATP utilization 
(ATPases). Thus, limitations imposed by the diffusion of ATP, and in particular ADP, are overcome by $\mathrm{Cr}$ and $\mathrm{PCr}$ with much higher diffusion rates and present in a combined concentration, for instance in muscle, up to six times higher than that of the adenine nucleotide pool (Wallimann et al. 2011). Mitochondrial CK in the mitochondrial outer compartment is tightly coupled to ATP export via adenine nucleotide carrier (ANT) and thus ATP-synthesis and respiratory chain activity, releasing $\mathrm{PCr}$ into the cytosol. The, extra-mitochondrial $\mathrm{Cr}$ concentration and the $\mathrm{PCr} / \mathrm{Cr}$ ratio are important kinetic parameters in the regulation of respiration and energy fluxes in muscle cells (Guzun et al. 2011).

While effects on the ergogenic role of $\mathrm{Cr} / \mathrm{PCr}$ may account for many of the beneficial effects of $\mathrm{Cr}$ supplementation now being reported in a number of degenerative diseases and neurological conditions, other potential roles have been noted. For instance, it has been reported that $\mathrm{Cr}$ exerts a direct antioxidant activity in cultured mammalian cells acutely injured by an array of free radical generating compounds, and importantly protects mtDNA and RNA from oxidative damage (Sestili et al. 2011). While the antioxidant effect exerted by $\mathrm{Cr}$ may be lower than other natural, plant, antioxidants, it is ubiquitous in many cells and therefore "physiologically on-board" (Sestili et al. 2011). In addition, $\mathrm{PCr}$ appears to be capable of stabilizing cellular membranes due to electrostatic interactions with phospholipids (Guzun et al. 2011). In cardiac cells, this helps in protecting the sarcolemma against ischemic damage, and $\mathrm{PCr}$ administered intravenously to patients with heart failure has been reported to improve their clinical condition (Guzun et al. 2011). Thus, the pleiotropic benefits of $\mathrm{Cr}$ in health, medicine and sport may be underpinned by a number of different mechanisms.

There is a growing body of literature demonstrating that $\mathrm{Cr}$ has a marked neuroprotective effect both in vitro and in vivo. As reviewed by Beal (2011), Cr supplementation extended survival, improved motor performance and reduced the loss of motor neurons in a transgenic mouse model of amyotrophic lateral sclerosis (ALS); and further, improved survival and motor function in a transgenic mouse model of Huntington's disease (HD). Due to its neuroprotective effects, $\mathrm{Cr}$ is today being investigated in clinical trials for the treatment of Parkinson's disease (PD) and HD (Beal 2011). As aging and neuro-degeneration share common pathophysiological pathways, Klopstock et al. (2011) investigated the effect of $\mathrm{Cr}$ supplementation on aging in wildtype mice. The median healthy life span of $\mathrm{Cr}$-fed mice was increased and they performed significantly better in neurobehavioral tests. Brains of Cr-treated mice showed evidence of reduced formation of free radicals and significantly lower accumulation of the "aging pigment" lipofuscin. An up-regulation of genes implicated in neuronal growth, neuroprotection, and learning further supported that $\mathrm{Cr}$ improved health and longevity in mice. Creatine may therefore be a promising food supplement to improve aging also in healthy humans (Klopstock et al. 2011).

Brain is the main organ affected in creatine-deficiency diseases arising from the absence of $\mathrm{Cr}$ synthesizing enzymes or expression of the $\mathrm{Cr}$ transporter (Braissant et al. 2011). Patients show delayed neurological development presenting with symptoms from early infancy. Today, it is known that $\mathrm{Cr}$ may cross the blood-brain barrier, albeit only with poor efficiency and that CNS must ensure parts of its $\mathrm{Cr}$ needs are met by endogenous synthesis. However, as discussed by Braissant et al. (2011), in many brain structures the enzymes of $\mathrm{Cr}$ synthesis, while found in every brain cell type, are not necessarily co-expressed, but expressed in a dissociated manner. As noted, the new understanding of $\mathrm{Cr}$ metabolism and transport in CNS will not only allow a better comprehension of the consequences of $\mathrm{Cr}$ deficiency in the brain, but will also help in delineating the physiological role of $\mathrm{Cr}$ in CNS.

Aging and reduced physical activity are associated with decreases in muscle mass, bone mineral density, and strength (Rawson and Venezia 2011; Tarnopolsky 2011; Wallimann et al. 2011). However, there is increasing evidence that $\mathrm{Cr}$ supplementation may slow and even reverse some of these changes. Recent studies have shown that in older adults, short-term high-dose $\mathrm{Cr}$ supplementation, independent of exercise training, may increase muscle strength and the capacity for physical exercise. Still greater benefits may be possible through the concurrent use of $\mathrm{Cr}$ supplementation with resistance training. Subsequent studies have also demonstrated that cognitive processing, that is either temporarily (following sleep deprivation) or permanently (due to aging) impaired, can be improved with $\mathrm{Cr}$ supplementation. The benefits afforded to older adults through $\mathrm{Cr}$ ingestion appear substantial, and ultimately may reduce the disease burden associated with sarcopenia and cognitive dysfunction (Rawson and Venezia 2011).

In theory, $\mathrm{Cr}$ supplementation could have a number of beneficial effects for myopathy patients including increases in muscle mass, strength and endurance capacity (Tarnopolsky 2011). As reported at the conference, patients with muscular dystrophy respond well to several months of $\mathrm{Cr}$ supplementation with gains in strength and fat-free mass. However, patients with myotonic dystrophy do not show a similar consistent effect. Creatine supplementation shows modest benefits only at lower doses in McArdle's disease patients, and may cause cramping at high doses. Patients with MELAS syndrome show some benefit from $\mathrm{Cr}$ supplementation in exercise capacity. The evidence for side effects or negative impact upon serological measures of $\mathrm{Cr}$ supplementation in all groups of myopathy patients is 
almost non-existent in comparison with the well-known side effects from current chemotherapeutic interventions (Tarnopolsky 2011).

Since the introduction of $\mathrm{Cr}$ supplementation procedures in the early 1990s, numerous allegations of adverse effects have been released through press media and scientific publications (Kim et al. 2011). Anecdotal reports from athletes have appeared on muscle cramp and gastrointestinal complaints. However, the incidence of these is limited and not necessarily linked to $\mathrm{Cr}$ itself. Despite several (unproved) allegations, liver enzymes, urea, and kidney glomerular filtration and albumin excretion rates show no change in young and healthy subjects supplemented for several months with $\mathrm{Cr}$. The potential production of mutagenic and carcinogenic heterocyclic amines by $\mathrm{Cr}$ supplementation was claimed by a French Sanitary Agency (AFSSA). However, formation of these is known to occur principally at high temperatures, such as in the searing of meat. Nonetheless, their presence in blood and urine following $\mathrm{Cr}$ supplementation should be looked for even if just to eliminate this concern. There is a slight increase (within the normal range) of urinary methylamine and formaldehyde excretion after a high dose of $\mathrm{Cr}$, although this is reduced when the dose is spread throughout the day (Kim et al. 2011). The most commonly used form of $\mathrm{Cr}$ is creatine monohydrate (CrM) (Jäger et al. 2011). Studies have shown that little or no $\mathrm{CrM}$ is degraded during normal digestion and that nearly $99 \%$ of orally administered CrM is either taken up by muscle or excreted in urine. Despite this, and the absence of medically significant side effects, manufacturers have continually sought to introduce other forms of $\mathrm{Cr}$. These newer forms have been purported to have better physical and chemical properties, bioavailability, efficacy, and/or safety profiles than CrM. However, there is no evidence that any of these are more effective and/or safer than CrM, whether ingested alone and/or in combination with other nutrients (Jäger et al. 2011). Furthermore, the regulatory position of these newer forms is uncertain in many countries, whereas that for CrM is clear.

Our understanding of $\mathrm{Cr}$ has clearly come a long way in the past century when in the 1920s, it was speculated to be a nitrogen store for protein synthesis in the body. However, for the most part we have overlooked in our debate on $\mathrm{Cr}$ that for much of hominid evolution this was a normal component of the diet and at levels comparable with those when $\mathrm{Cr}$ supplements are taken (Wallimann et al. 2011). Indeed, on a $\mathrm{mg} / \mathrm{kg}$ body weight basis, the "high" levels taken by athletes when "Cr loading" are close to and even surpassed by those ingested by any carnivorous animal. It may be that we should be regarding such "high" levels as closer to the normal level for humans, rather than the very low levels found in modern diets (as low as $0 \mathrm{~g}$ in the vegan diet). One to two grams of $\mathrm{Cr}$ is minimally required per day by the adult human and this is clearly an "expensive" compound for the body to make. It is little wonder, therefore, that dietary sources of $\mathrm{Cr}$, when available, are utilized, whereas this is not the case in at least some herbivores (Sewell and Harris 1995). Arginine, one of two amino acid precursors of $\mathrm{Cr}$, is required also in the urea cycle, and for nitric oxide and protein synthesis. Although not classified as an essential amino acid, diets with a low protein intake, such as in the elderly where "economic anorexia" is an issue, will increase the burden placed on this by $\mathrm{Cr}$ synthesis (Brosnan et al. 2011). In addition, daily $\mathrm{Cr}$ synthesis consumes a major fraction of the labile methyl groups provided by S-adenosylmethionine (Brosnan et al. 2011).

While it is evident that humans can function in the absence of a dietary supply of $\mathrm{Cr}$, there are likely to be instances, for example in the elderly, those on a protein restricted diet, vegans, pregnant and lactating women, and in the premature and new born (Wallimann et al. 2011) where consideration perhaps should be given to inclusion of $\mathrm{Cr}$ in the diet. Certainly, this would seem to be the case for the otherwise-healthy elderly population where age progression, and age-related changes, for example in bone mineral density appear to benefit from $\mathrm{Cr}$ supplementation (Wallimann et al. 2011). Such health related benefits, affecting individuals as well as national health budgets, may be realized from a compound which could almost be made available free of charge. As reviewed in this special issue of Amino Acids, there is increasing evidence for beneficial and clinically significant effects in neurological disorders and myopathies, where current drug treatments are both many times more expensive and associated with side effects. This contrasts to $\mathrm{Cr}$ which is both a normal nutrient and seemingly free of any deleterious effects. Sadly, there is little scope for major financial gains to be made from $\mathrm{Cr}$, and therefore little incentive for major companies to fund large scale clinical trials.

At the root of any health or medical benefits lies the nature of the pleiotropic actions of $\mathrm{Cr}$ itself, from maintenance of intracellular adenine nucleotide homeostasis, the stimulation of mitochondrial respiration and transduction through the cell of high energy phosphate units, to other less well defined functions such as its antioxidant activity. Many more cell types are recognized today as having a $\mathrm{Cr} / \mathrm{PCr} / \mathrm{CK}$ system than was previously thought, and it is to be expected that more effects of $\mathrm{Cr}$ elevation in these will be described in the future.

Guest of Honour at the Downing College meeting was Professor Eric Hultman who over 40 years had made many unique contributions to research on $\mathrm{Cr}$ in humans. Sadly, Professor Hultman died in March of this year, just prior to the release of this special issue. His work and the many contributions he made will be remembered long into the future. 


\section{References}

Beal MF (2011) Neuroprotective effects of creatine. Amino Acids 40(5):1305-1313

Braissant O, Henry H, Béard E, Uldry J (2011) Creatine deficiency syndromes and the importance of creatine synthesis in the brain. Amino Acids 40(5):1315-1324

Brosnan JT, da Silva RP, Brosnan ME (2011) The metabolic burden of creatine synthesis. Amino Acids 40(5):1325-1331

Guzun R, Timohhina N, Tepp K, Gonzalez-Granillo M, Shevchuk I, Chekulayev V, Kuznetsov AV, Kaambre T, Saks VA (2011) Systems bioenergetics of creatine kinase networks: physiological roles of creatine and phosphocreatine in regulation of cardiac cell function. Amino Acids 40(5):1333-1348

Hultman E, Bergstrom J, McLennan Anderson N (1967) Breakdown and resynthesis of phosphorylcreatine and adenosine triphosphate in connection with muscular work in man. Scand J Clin Lab Invest 19:56-66

Hultman E, Söderlund K, Timmons JA, Cederblad G, Greenhaff PL (1996) Muscle creatine loading in men. J Appl Physiol 81:232-237

Jäger R, Purpura M, Shao A, Inoue T, Kreider RB (2011) Analysis of the efficacy, safety, and regulatory status of novel forms of creatine. Amino Acids 40(5):1369-1383
Kim HJ, Kim CK, Carpentier A, Poortmans JR (2011) Studies on the safety of creatine supplementation. Amino Acids 40(5):1409-1418

Klopstock T, Elstner M, Bender A (2011) Creatine in mouse models of neurodegeneration and aging. Amino Acids 40(5):1297-1303

Rawson ES, Venezia AC (2011) Use of creatine in the elderly and evidence for effects on cognitive function in young and old. Amino Acids 40(5):1349-1362

Sahlin K, Harris RC (2011) The creatine kinase reaction: a simple reaction with functional complexity. Amino Acids 40(5):1363-1367

Sestili P, Martinelli C, Colombo E, Barbieri E, Potenza L, Sartini S, Fimognari C (2011) Creatine as an antioxidant. Amino Acids 40(5):1385-1396

Sewell DA and Harris RC (1995) Effect of creatine supplementation in the Thoroughbred horse. Equine Exercise Physiology 4. Equine Vet J Suppl 18:239-242

Tarnopolsky MA (2011) Creatine as a therapeutic strategy for myopathies. Amino Acids 40(5):1397-1407

Wallimann T, Tokarska-Schlattner M, Schlattner U (2011) The creatine kinase system and pleiotropic effects of creatine. Amino Acids 40(5):1271-1296 